I N S T I T U T O

DE

M E D I C I N A

T R O P I C A L

DE

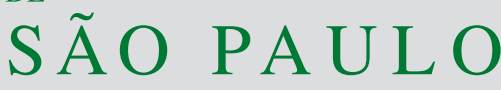

JOURNAL OF THE SÃO PAULO INSTITUTE OF TROPICAL MEDICINE

'Universidade Federal do Maranhão, Programa de Pós-Graduação em Ciências da Saúde, São Luís, Maranhão, Brazil

${ }^{2}$ Universidade Federal do Maranhão, Departamento de Biologia, Laboratório de Genética e Biologia Molecular, São Luís, Maranhão, Brazil

${ }^{3}$ Universidade Federal do Maranhão Departamento de Biologia, Laboratório de Entomologia e Vetores, São Luís, Maranhão, Brazil

${ }^{4}$ Universidade Paulista, Instituto de Ciências da Saúde, Bauru, São Paulo, Brazil

Correspondence to: Rosa Cristina Ribeiro da Silva

Universidade Federal do Maranhão, Departamento de Biologia, Laboratório de Genética e Biologia Molecular, Cidade Universitária Dom Delgado, CEP 65080805 , São Luís, MA, Brazil

Tel: +559832728543

E-mail: rosinhachris@gmail.com

Received: 15 July 2021

Accepted: 21 September 2021

\section{Experimental transmission of Leishmania (Leishmania) amazonensis to immunosuppressed mice through the bite of Lutzomyia longipalpis (Diptera: Psychodidae) results in cutaneous leishmaniasis}

\author{
Rosa Cristina Ribeiro da Silva ${ }^{(1,2,3}$, Léo Nava Piorsky Dominici Cruz ${ }^{(2,3}$, \\ João Manoel da Silva Coutinho ${ }^{\circledR, 3}$, Carlos Eduardo Fonseca-Alves ${ }^{\circledR}$, \\ José Manuel Macário Rebêlo ${ }^{\circledR 3}$, Silma Regina Ferreira Pereira ${ }^{\circledR}$
}

\section{ABSTRACT}

Lutzomyia longipalpis is the natural vector of Leishmania (Leishmania) infantum, but it is also permissive for several Leishmania species that are related to cutaneous leishmaniasis (CL). Maranhao State (Northeast of Brazil) is endemic for CL and has the highest number of cases of diffuse cutaneous leishmaniasis (DCL) in the country. It is a rare disease associated with a defective immune response mainly caused by $L$. (L.) amazonensis. Additionally, the number of immunosuppressed patients infected with the etiologic agents of CL has increased, including regions in which the main vectors of $\mathrm{CL}$ are rare. Therefore, we investigated whether Lu. longipalpis is able to transmit $L$. (L.) amazonensis to uninfected and immunosuppressed mice, resulting in CL. For that, 291 sand flies took an initial blood meal in mice infected with $L$. (L.) amazonensis. Of these, 17 underwent a second feeding on uninfected and immunosuppressed mice (of which $58.8 \%$ were also positive for Leishmania according to data on the dissection of the intestine). After 27 days of infection, these mice exhibited leishmaniotic lesions. The occurrence of parasites on the animal's skin was confirmed by limiting dilution and immunohistopathological analyses. Parasite DNA was also detected in paw lesions and inguinal lymph nodes. DNA sequencing confirmed the Leishmania species in insects and mice. The results confirmed the ability of $L u$. longipalpis to become infected and experimentally transmit $L$. (L.) amazonensis to immunosuppressed rodents, resulting in leishmaniotic lesions. Our data open perspectives for the potential role of Lu. longipalpis in the epidemiology of urban cutaneous leishmaniasis, especially in immunosuppressed patients.

KEYWORDS: Cutaneous leishmaniasis. Experimental infection. Host-parasite interaction. Permissive vector. Vector 1 competence.

\section{INTRODUCTION}

Lutzomyia longipalpis (Diptera, Psychodidae) is the most relevant vector of Leishmania (Leishmania) infantum (Kinetoplastida, Trypanosomatidae), the etiological agent of visceral leishmaniasis (VL) in the New World ${ }^{1}$. Nevertheless, natural infection ${ }^{2,3}$ and experimental infection studies ${ }^{4,5}$ have highlighted the ability of Lu. longipalpis to sustain the development of other species causing cutaneous leishmaniasis (CL).

In the American continent, CL is caused by several species of Leishmania, among which L. (Viannia) braziliensis and L. (L.) amazonensis are the most prevalent in Brazil ${ }^{6}$. In the Northeast of the country, Maranhao State is endemic 
to $\mathrm{CL}$ and has the highest number of diffuse cutaneous leishmaniasis (DCL), a rare disease associated with a defective immune response mainly caused by $L$. (L.) amazonensis, whose vector is Bichromomyia flaviscutellata ${ }^{7}$. Moreover, other studies have described the high prevalence of Leishmania spp. infections in immunosuppressed people ${ }^{8,9}$, including $\mathrm{HIV} / \mathrm{CL}^{10}$ and HIV/DCL ${ }^{11}$ coinfections in Maranhao State.

Studies have described the spread of CL in urban environments ${ }^{12,13}$ in which Lu. longipalpis feeds on several domestic and synanthropic animals ${ }^{2,14}$, showing a higher adaptive capacity to seasonal variations than other sand flies species ${ }^{15}$. Additionally, studies have reported the circulation of several species of Leishmania, including $L$. (L.) amazonensis, concomitantly with Lu. longipalpis in $\mathrm{Brazil}^{2,3}$. These facts have generated the hypothesis that $\mathrm{Lu}$. longipalpis may be infected and be able to transmit L. (L.) amazonensis, mainly in areas where its natural vectors are rare $^{12-17}$.

Despite this permissiveness, there are no studies regarding the ability of $\mathrm{Lu}$. longipalpis to experimentally transmit $L$. (L.) amazonensis to mice, in the same way it was investigated for $L$. (V.) braziliensis ${ }^{18}, L$. (L.) mexicana ${ }^{19}$ and $L$. (L.) major ${ }^{20}$. Therefore, taking into account the occurrence of $\mathrm{CL}$ in immunocompromised patients, our study aimed to test the hypothesis that $\mathrm{Lu}$. longipalpis has the capacity to transmit $L$. (L.) amazonensis to immunosuppressed rodents, resulting in cutaneous lesions.

\section{MATERIALS AND METHODS}

\section{Sand flies, parasite and mice}

Specimens of $L u$. longipalpis were reared in the laboratory, free from Leishmania infection, as previously described $^{21}$. Twelve hours before the experiments, the feeding of female sand flies was suspended to increase the probability of the insects feeding on the mice infected by $L$. (L.) amazonensis (IFLA/BR/1968/PH8 strain). Promastigotes were grown in Schneider medium (Sigma, St Louis, MO, USA), supplemented with $1 \%$ antibiotic (Penicillin $100 \mu \mathrm{L} / \mathrm{mL}$ and streptomycin $50 \mu \mathrm{L} / \mathrm{mL}$ ) and $20 \%$ inactivated bovine fetal serum (GIBCO), and kept in an incubator at $27^{\circ} \mathrm{C}$.

Balb/c mice (Mus musculus, $25 \mathrm{~g}$ ) were obtained from the Vivarium of the University of Campinas, Sao Paulo, Brazil, under pathogen-free conditions. The animals were kept in polycarbonate boxes under controlled conditions: temperature $21 \pm 2{ }^{\circ} \mathrm{C}$ and an alternating light-dark cycle of $12 \mathrm{~h}$. This study was approved by the Ethics Committee on Animal Experimentation/UFMA (23115.006003/2017-11).

\section{Experimental infection of mice for the $1^{\text {st }}$ blood meal}

Eight male Balb/c mice, aged 15 weeks, were experimentally infected in the right hind paw by inoculation with $1 \times 10^{7}$ promastigotes of $L$. (L.) amazonensis in the stationary phase, as previously described ${ }^{22}$. On the $41^{\text {st }}$ day after infection, mice were anesthetized $(300 \mathrm{mg} / \mathrm{kg}$ of ketamine and $60 \mathrm{mg} / \mathrm{kg}$ of xylazine) to feed the female sand flies (three to four days of age) emerging from the insect colony. The animals were placed in nylon and glass-lined cages, with metal frames $(30 \times 30 \times 30 \mathrm{~cm})$, placed in a biochemical oxygen demand (BOD) oven, in the dark, at a temperature of $27^{\circ} \mathrm{C}$ and a relative humidity of $80 \%$.

\section{Immunosuppression of mice for the $2^{\text {nd }}$ blood meal}

Four uninfected male Balb/c mice, aged 35 weeks and weighing $28 \mathrm{~g}$, had their susceptibility to infection increased by the administration of a single dose of cyclophosphamide ( $50 \mathrm{mg} / \mathrm{kg} /$ mouse) intraperitoneally ${ }^{23}$. After $48 \mathrm{~h}$, the animals were anesthetized and subjected to the exposure to sand fly bites following the same protocol described above.

\section{Experimental design}

Firstly, infection experiments (called ExpA) corresponding to the infection of Lu. longipalpis by $L$. (L.) amazonensis were carried out by the biting of experimentally infected mice ( $1^{\text {st }}$ blood meal). The tests were carried out according to the number of available females emerging from the insect colony, totaling 485 females with three to four days of life, from the F1, F2, and F3 generations. Eight infected Balb/c mice were placed inside a cage to serve as a food source for sand flies, so that two animals were exposed to the same group of sand flies, sequentially, for 60 min each. Each group ( 27 to 65 females/animal) had a total of $120 \mathrm{~min}$ available to feed, totaling 12 blood meal attempts (groups I-XII). Supplementary Figure S1A shows the experimental insect infection scheme.

The transmission experiment (called ExpB) refers to the transmission of $L$. (L.) amazonensis to an uninfected mouse through the bite of Lu. longipalpis from ExpA during the insects' second blood meal (Supplementary Figure S1B). ExpB was performed 5 to 10 days after ExpA by offering an immunosuppressed and infection-free animal as a food source for insects, for $60 \mathrm{~min}$. Among the insects fed on four animals during 12 attempts, two survived for at least two months after the feeding. The two surviving animals were named Animal A and Animal B. 
All engorged females from the first or second feeding were investigated for the presence of the parasite by midgut dissection and Leishmania DNA detection. The experimental infection rate was considered as the number of positive females for Leishmania in relation to the total number of engorged females. Supplementary Table S1 displays all groups used in each assay and the rates of engorged females, experimental infection rates and animal outcomes after being a food source for sand flies.

\section{Dissection of engorged Lutzomyia longipalpis}

All females $(\mathrm{N}=17)$ that underwent the second blood meal were dissected to assess the presence or absence of $L$. (L.) amazonensis promastigotes in the intestine. From 6-10 days after the second meal, females were anesthetized for $5 \mathrm{~min}$ at $-20^{\circ} \mathrm{C}$ and individually dissected, exposing the intestine using drops of $0.9 \% \mathrm{NaCL}$ to observe the presence of promastigote forms under an optical microscope.

\section{Leishmania (L.) amazonensis DNA confirmation by polymerase chain reaction and sequencing}

Engorged females $(\mathrm{N}=291)$ and samples of the mice used in the transmission test (lesion of the paw, spleen, liver and inguinal lymph node) had DNA extracted for the detection of Leishmania DNA by amplifying a 300 to 350 base pair (bp) fragment of the ITS- 1 gene, using the primers LITSR (5'-CTGGATCATTTTCCGATG-3') and L5.8S (5'-TGATACCACTTATCGCACTT-3') according to a previous study ${ }^{24}$. Amplified DNA was purified using ExoSAP-IT PCR Product Cleanup Reagent; (Thermo Fisher Scientific ${ }^{\mathrm{TM}}$, Waltham, USA), and the DNA sequencing was performed by the company ACT Gene Analises Moleculares Ltda. The sequences were deposited on the GenBank platform of the National Center for Biotechnological Information, under the access numbers available in Supplementary Tables S2 and S3. The sequences obtained were aligned with ITS- 1 sequences, and the phylogeny reconstruction was performed following previous studies $^{11,25}$.

\section{Determination of parasitic load}

To confirm that $L$. (L.) amazonensis was transmitted via $L u$. longipalpis, mice were examined weekly for their general physical condition (shedding of hair and changes in skin condition) in addition to the appearance of skin lesions. When there were clinical signs suggestive of infection, the mouse was euthanized by anesthetic overdose $(60 \mathrm{mg} / \mathrm{kg}$ of xylazine and $300 \mathrm{mg} / \mathrm{kg}$ of ketamine), and spleen, liver, lymph nodes and skin lesions of the paw and snout were collected for the investigation of the presence of the parasite.

The parasitic loads of the spleen, liver, inguinal lymph nodeand foot injuries were determined using the limiting dilution method ${ }^{26}$. This assay was performed in duplicate and incubated at $27{ }^{\circ} \mathrm{C}$. After 24,48 and $72 \mathrm{~h}$, the samples from each well were analyzed and defined as positive or negative, depending on the presence or absence of promastigotes in each well. The total number of parasites/ samples was calculated as previously described ${ }^{27}$.

\section{Histopathological and immunohistochemistry analysis}

Fragments of spleen, liver and skin lesion of the left paw were collected, fixed in $10 \%$ formaldehyde for $24 \mathrm{~h}$, later processed and embedded in pure paraffin. Subsequently, 5- $\mu \mathrm{m}$ sections were prepared and the slides were stained using hematoxylin and eosin and Giemsa techniques. Histological sections were observed under a light microscope (40 X magnification).

The immunohistochemical analysis was performed to identify amastigotes in the skin, spleenand liver, as previously described ${ }^{28}$. Briefly, serum from a dog naturally infected by $L$. (L.) amazonensis was used as a primary antibody, and a polyclonal goat anti-mouse was used as a secondary antibody. The immunohistochemistry assay was stained with 3 3'-diaminobenzidine, and tissue samples were counterstained with Harris' hematoxylin. The immunohistochemistry analysis was considered positive when amastigotes were visualized stained in brown color. In this manner, samples were scored as positive or negative. We used spleen samples from a non-infected mouse and samples with amastigotes identified by histology as the negative and the positive control, respectively.

\section{The proportion of blood-feeding and mortality of Lutzomyia longipalpis}

We calculated the proportion of females who performed blood feeding (PBF) from the first blood meal (mouse infected by Leishmania inoculation) and the second blood meal (naive mouse). The mortality proportion (PM) was calculated as the number of engorged females killed after the first blood meal in relation to the total number of females that were engorged, dead, or survived ${ }^{14}$. The differences between the first and second feeding proportions were analyzed using the non-parametric Mann-Whitney test $(\mathrm{p}<0.05)$. 


\section{RESULTS}

\section{Proportion of blood-feeding and mortality of Lutzomyia longipalpis}

We used 485 female sand flies in the infection tests distributed in groups I-XII, which were exposed to feed on eight mice infected with $L$. (L.) amazonensis (ExpA). Of these, 291 (0.60) took the first blood meal, of which 146 $(50 \%)$ survived and were exposed to the second feeding in the uninfected animal (ExpB), with a blood meal proportion of 0.12 (17), and a significant difference in the proportion of first blood meal $(p=0.0001)(\operatorname{ExpB})($ Table 1$)$. The female sand flies were preferably fed on the mice's snouts and feet; however, feeding was sporadically observed on the animal's tail, earsand eyelids.

\section{Experimental infection of sand flies by Leishmania (L.) amazonensis}

Of the 291 females of Lu. longipalpis that took the first blood meal in a mouse infected with $L$. (L.) amazonensis, we identified Leishmania DNA in 153 insects (infection rate $=52.6 \%$; Table 2, Figure 1A). Additionally, 21 females were dissected after the blood meal, of which ten $(47.6 \%)$ had flagellate forms of the parasite in their intestines (Table 2).
Transmission of Leishmania (L.) amazonensis to mice by Lutzomyia longipalpis

For this analysis, we considered only the groups IX, $\mathrm{XI}$ and XII, whose females had made the first blood meal $(\mathrm{N}=74)$, of which seven were able to perform the second blood meal on two uninfected mice that survived for at least, two months after the bites (called animal A and animal B). We observed promastigotes in 4/7 of these sand flies.

Animal A: Eight days after the first blood-feeding, 22 surviving insects were subjected to a second blood meal in animal A. Of the 22 females, only two insects performed the second feeding in this mouse (Table 3), one of which was positive for the presence of Leishmania promastigotes. Nevertheless, after 96 days of observation of the animal, there were no clinical signs of disease. This animal was euthanized, and the limiting dilution test revealed no promastigote forms, indicating no transmission of the parasite to Animal A.

Animal B: This animal was subjected twice, at different times, to the second blood-feeding of sand flies (in groups XI and XII):

In group XI, after seven days of the first blood-feeding, 36 females were subjected to a second blood meal in animal $\mathrm{B}$, and only three females $(8 \%)$ were able to perform the second feeding, of which $2 / 3$ were later confirmed as positive by intestine dissection and polymerase chain reaction (PCR) (Table 3).

Table 1 - Proportions of engorged females and proportion of mortality of Lutzomyia longipalpis females that performed blood feeding on mice, according to the first (ExpA) and the second (ExpB) blood meals.

\begin{tabular}{|c|c|c|c|c|c|c|c|c|}
\hline \multirow{2}{*}{ Group } & \multicolumn{5}{|c|}{ ExpA: first blood meal } & \multicolumn{3}{|c|}{ ExpB: second blood meal } \\
\hline & TF & $\mathrm{EF}$ & PBF & TFD & PM & TF & $\mathrm{EF}$ & PBF \\
\hline $\mathrm{I}$ & 38 & 11 & 0.29 & 7 & 0.64 & 4 & 3 & 0.75 \\
\hline II & 41 & 9 & 0.22 & 6 & 0.67 & 3 & 1 & 0.33 \\
\hline III & 29 & 6 & 0.21 & 0 & - & 6 & 1 & 0.17 \\
\hline IV & 37 & 11 & 0.30 & 5 & 0.45 & 6 & 0 & - \\
\hline $\mathrm{V}$ & 65 & 34 & 0.52 & 23 & 0.68 & 11 & 0 & - \\
\hline $\mathrm{VI}$ & 50 & 28 & 0.56 & 11 & 0.39 & 17 & 0 & - \\
\hline VII & 57 & 40 & 0.70 & 26 & 0.65 & 14 & 5 & 0.36 \\
\hline VIII & 32 & 31 & 0.97 & 20 & 0.65 & 11 & 0 & - \\
\hline IX & 30 & 27 & 0.90 & 5 & 0.19 & 22 & 2 & 0.09 \\
\hline$x$ & 27 & 19 & 0.70 & 19 & 1.00 & 0 & 0 & - \\
\hline $\mathrm{XI}$ & 51 & 48 & 0.94 & 12 & 0.25 & 36 & 3 & 0.08 \\
\hline XII & 28 & 27 & 0.96 & 11 & 0.41 & 16 & 2 & 0.13 \\
\hline Total & 485 & 291 & 0.60 & 145 & 0.50 & 146 & 17 & 0.12 \\
\hline Mean (SD) & & & $0.61(0.27)$ & & $0.54(0.21)$ & & & $0.25(0.22)$ \\
\hline
\end{tabular}

TF = Total females exposed to feed on mice; EF = Engorged females; PBF = Proportion of blood-feeding; TFD = Total Females dead after the first blood meal; $\mathrm{PM}=$ Proportion of mortality of the first blood meal; SD = Standard deviation. 
Table 2 - Experimental infection rate and number of dissected Lutzomyia longipalpis females exposed to the first blood meal (ExpA) on mice infected with Leishmania (Leishmania) amazonensis.

\begin{tabular}{lcccc}
\hline Group & Engorged females & *Intestine dissection (+) & ${ }^{* *}$ PCR & \#Infection rate (\%) \\
\hline I & 11 & $3(3)$ & 6 & 54.5 \\
II & 9 & $1(0)$ & 7 & 77.8 \\
III & 6 & $1(1)$ & 11 & 66.7 \\
IV & 11 & 0 & 11 & 100.0 \\
V & 34 & $2(0)$ & 23 & 32.4 \\
VI & 28 & 0 & 19 & 82.1 \\
VII & 40 & $5(2)$ & 4 & 47.5 \\
VIII & 31 & $1(1)$ & 20 & 12.9 \\
IX & 27 & $2(1)$ & 10 & 74.0 \\
X & 19 & 0 & 20 & 52.6 \\
XI & 48 & $3(2)$ & 18 & 42.0 \\
XII & 27 & $3(1)$ & 153 & 52.0 \\
Total & 291 & $21(10)$ & 52.6 \\
\hline
\end{tabular}

*Number of females, whose intestine was dissected to investigate the presence of Leishmania; (+)-Number of females, in which the parasite was observed in the intestine; ${ }^{* *} \mathrm{PCR}$ : positive Polymerase Chain Reaction for Leishmania; \# Calculated through the number of females positive for Leishmania DNA in relation to the number of engorged females.

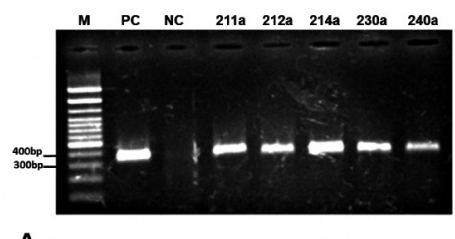

A

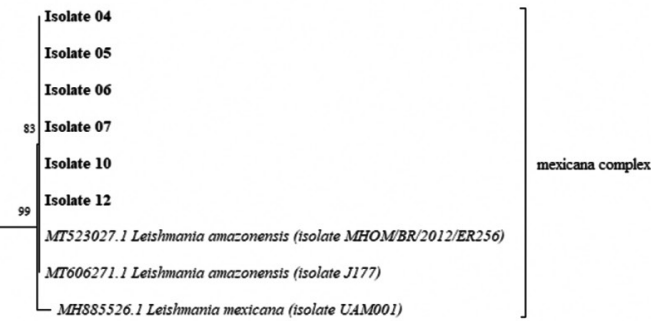

96 G0 GU045591.1 Leishmania infatum (isolate 20 clone 1)

85 FR675940.1 Leishmania infantun (strain MHOMUS/10/SLRHCLI)

AJ634378.1 Leishmania donovant (isolate MHOMIN/OL/BHU20140)

.6 AM901453.1 Leishmania donovani (strain MCAN/MA/2002/AD3)

37 HG512923.1 Leishmania aethiopica (strain MHOMET/83/85-83 [LEM1636])

${ }_{94}$ HG512962.1 Leishmania major (strain MHOM/SN/2004/ICB39 [LEM4797])

47 HG512963.1 Leishmania major (strain MHOMBFF/2004/RENO4-S [LEM48S6])

90 AS000309.1 Leishmania turanica (strain MRHO/MN/84/MNR13)

98 AJ272379.1 Leishmania turanica (strain M103)

HG512959.1 Leishmania panamensis (strain MHOMCR/2004/TIM13 [LEM4743])

FN398331.1 Leishmania givanensis (strain MHON/BR/2002/NMT-RBO013)

99 FN398340.1 Leishmania perviviana (strain MHOMPE/2006/LH3667)

FN398337.1 Letshmania braziliensis (strain MHOMPE/2003/LH2920)

63 JQ061322.1 Leishmania braziliensis (isolate MHOMBR/2006/EFSF)

AF362831.1 Tiypanosoma criet (isolate Ro)

B

Figure 1 - Confirmation of Leishmania amazonensis DNA in Lutzomyia longipalpis fed on infected mice (A): $1 \%$ agarose gel showing amplification products of 300-350 bp generated by primers from Leishmania ITS1 sequence . M = 100 pb marker; PC: positive control; NC: negative control; 211a - 240a: positive samples from sand flies infected with Leishmania (Leishmania) amazonensis. (B) Evolutionary scenario of ITS-1 gene inferred by the Neighbor-Joining method. The percentage of replicate trees in which the associated taxa clustered together in the bootstrap test (1,000 replicates) is shown next to the branches. The branch lengths are in the same units as those of the evolutionary distances used to infer the phylogenetic tree. Sequences from our study are highlighted in bold (isolates 04 to 07 refer to samples of sand flies infected with L. (L.) amazonensis; isolate 10 refers to the sample of the strain isolated from the lesion of animal $B$ and isolate 12 refers to the sample of the strain isolated from culture, the same as the positive control in Figure 1A). Brackets indicate sequences from the Mexican complex,including Leishmania amazonensis and Leishmania mexicana. Trypanosoma cruzi is the outgroup. 
Table 3 - Number of females of Lutzomyia longipalpis that underwent the second blood meal in two uninfected and immunosuppressed mice ( $\mathrm{A}$ and $\mathrm{B}$ ) and the total of females diagnosed as positive for Leishmania (Leishmania) amazonensis through direct observation of parasites by intestinal dissection and DNA detection by Polymerase Chain Reaction (PCR).

\begin{tabular}{lccccc}
\hline Group/animal & Incubation* $^{*}$ & $\begin{array}{c}\text { Females exposed to } \\
\text { the 2 }\end{array}$ & blood meal & Engorged females & \multicolumn{2}{c}{ Females infected with Leishmania } \\
\cline { 3 - 6 } IX/ Animal A & 8 & 22 & 2 & 1 & Intestine dissection \\
XI/ Animal B & 7 & 36 & 3 & 2 & 1 \\
XII/ Animal B & 6 & 16 & 2 & 1 & 1 \\
\hline Total & - & 74 & 7 & 4 & 4 \\
\hline
\end{tabular}

${ }^{*}$ Number of days after the $1^{\text {st }}$ blood meal.

In group XII, after six days of the first blood-feeding, a total of 16 females were subjected to a second meal in animal B, and only two (12.5\%) were successfully fed, of which $1 / 2$ was later confirmed to be positive by dissection and PCR (Table 3). In total, animal B was bitten by five females, of which three $(60 \%)$ were infected with Leishmania. The animal was observed for 67 days developing a lesion (slight swelling) in the left upper paw on the $27^{\text {th }}$ and $22^{\text {nd }}$ days after exposure to females from groups $\mathrm{XI}$ and XII, respectively. Figure 2 shows the progression of the lesion.

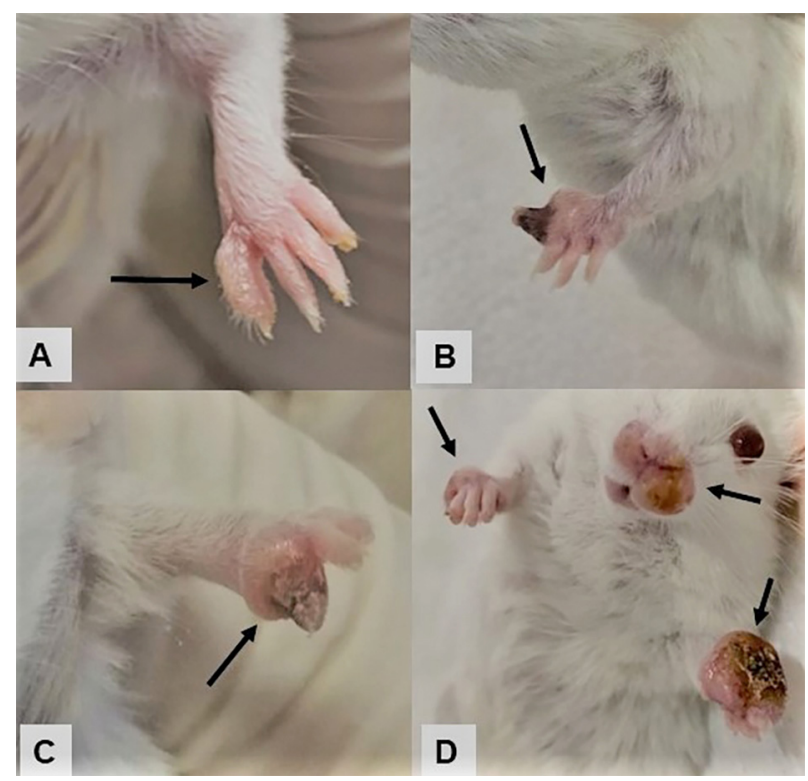

Figure 2 - Progression of the lesion observed in the mouse (animal B) bitten by Lutzomyia longipalpis and infected with Leishmania (Leishmania) amazonensis. In (A) 27 and 22 days after the bite; (B) 47 and 42 days; (C) 55 and 50 days; (D) 67 and 62 days concerning groups $\mathrm{XI}$ and $\mathrm{XII}$, respectively.

\section{Parasitic load and DNA sequencing}

Ten weeks after exposure to the bites of infected insects, animal B was euthanized. The limiting dilution test demonstrated the Leishmania spread from the paw (location of the insect bite) to the viscera (spleen, liver and inguinal lymph node). The parasitic load observed in the spleen was $\left.4.3 \times 10^{4}(0.1 \mathrm{~g} / \mathrm{mL}), 3.0 \times 10^{3}(0.3 \mathrm{~g}) / \mathrm{mL}\right)$ in the liver, and $8.9 \times 10^{3}(0.03 \mathrm{~g}) / \mathrm{mL}$ in the inguinal node. The lesion on the paw (origin of the insect bite) showed a higher parasitic load of $9.0 \times 10^{4}(0.1 \mathrm{~g} / \mathrm{mL})$.

To confirm the origin of the lesion as being caused by the bite of Lu. longipalpis infected by $L$. (L.) amazonensis, we carried out the DNA sequencing. The result of the $\mathrm{NJ}$ analysis (Figure 1B) confirmed the identity of ITS-1 sequences obtained from the total DNA of infected sand flies that took a second meal in animal B (isolates 4, 5, 6 and 7) from the lesion sample on the paw of animal B (isolate 10), and the sample of the positive control obtained from the cultured strain (isolate 12). All sequences belong to the same clade of the species $L$. (L.) amazonensis in the Mexican complex, indicating that they are the same parasite used in the study.

\section{Histopathology and immunohistochemistry}

In the histopathological analysis of the spleen, no morphological changes or the presence of amastigotes were observed in splenic macrophages. When evaluating the spleen samples using the Giemsa staining, no macrophages with Leishmania amastigote forms were identified. In the morphological analysis of the liver, we observed a slight degeneration of hepatocytes; there were no macrophages with amastigote forms observed in the morphological analysis. In the skin lesion analysis, we observed an intense neutrophilic inflammatory infiltrate with an area of necrosis and cellular apoptosis. There was an area of multifocal hemorrhage and collagenolysis, in addition to a moderate number of macrophages with Leishmania amastigote forms and extracellular amastigote forms in areas of cell degeneration (Figure 3A). We identified amastigote forms using hematoxylin-eosin and Giemsa stainings.

In the immunohistochemical analysis, no amastigote forms of Leishmania were observed in the liver and 

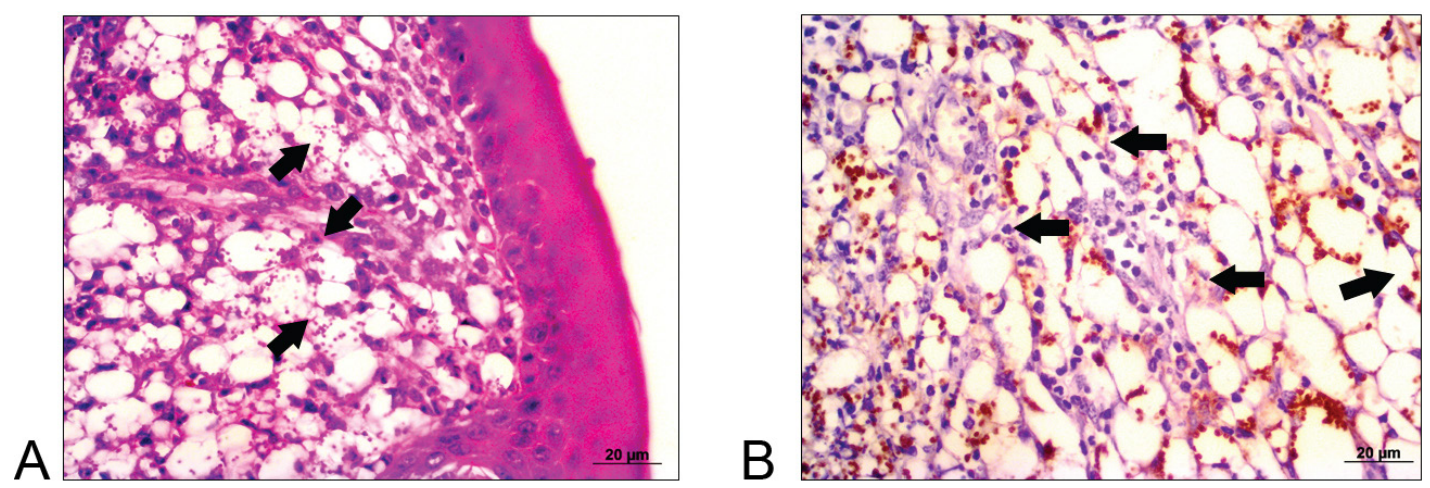

Figure 3 - Visualization of Leishmania in the mouse bitten by infected sand flies. (A) Hematoxylin and eosin staining of a skin lesion showing intense cell degeneration and several amastigotes (arrows) in the degenerate cell cytoplasm. $40 \mathrm{x}$ magnification. Scale bar: $20 \mu \mathrm{m}$. (B) Skin lesion showing several amastigotes stained in brown color, confirming the Leishmania infection origin for this lesion. Immunohistochemistry, Harris' hematoxylin counterstaining, 40 x magnification.

spleen samples. However, numerous forms of Leishmania amastigotes were observed in the skin lesion (Figure 3B). Several amastigotes were also observed in regions of degeneration, edema and cell necrosis.

\section{DISCUSSION}

In the Americas, from the Southern United States to Northern Argentina, with the exception of Chile, $L$. (L.) amazonensis is transmitted by B. faviscutellata ${ }^{1}$. In Brazil, this vector is rarely found in peridomicile of urban environments ${ }^{6,15,29}$, however $L$. (L.) amazonensis is found infecting $L u$. longipalpis in these areas, where this vector is highly adapted ${ }^{2}$. In this context and considering the increase in the number of patients coinfected with Leishmania and HIV, mainly in the Northeast of Brazil ${ }^{11,30,31}$, we evaluated the role of the sand fly Lu. longipalpis as a competent vector for $L$. (L.) amazonensis. To accomplish this, we carried out an experimental transmission assay of the parasite to an uninfected and immunosuppressed vertebrate mammal.

The Balb/c mouse was previously used as the sole blood-feeding host for female $\mathrm{Lu}$. longipalpis, proving to be a very attractive model, with PBF greater than 50\%.In natural conditions, these observations may be associated with the sand fly's eclectic eating behavior, displaying no food preference, and their ability to take a blood meal on any available vertebrate animal, including man ${ }^{2,14}$. Despite the PBF rate, more than half of the engorged females died before taking the second feeding. We observed that some females had a distended abdomen due to a large number of eggs. According to some authors, many matured eggs can cause stress and exhaustion, culminating in the death of females ${ }^{32}$. However, despite the high mortality, the surviving females were able to transmit the parasite.

Lu. longipalpis females have also presented high rates of infection by $L$. (L.) amazonensis (53\%). This finding demonstrates the high permissiveness of the species, which translates into the ability of this vector to sustain infection by $L$. (L.) amazonensis. This high rate of infection can be explained by the degree of cutaneous parasitism in the mouse because these animals were exposed to insects only after the appearance of leishmaniotic lesions (45 days after infection), increasing the probability of sand flies to become infected ${ }^{14}$.

Transmission studies are challenging because of the high mortality rate of insects after the first blood meal, with few specimens remaining to perform the second meal ${ }^{33}$. With a small number of females available for the second blood meal, high infection rates increased the chance of obtaining infected females surviving the first meal. Another factor that implies successful transmission is the state of the vertebrate host's immune system ${ }^{31}$. In this context, immunosuppression of mice may have been a factor that facilitated transmission, however, when the immunosuppressed animal A was subjected once to a second blood meal, it did not show any clinical signs of $\mathrm{CL}$, corroborating the difficulty of transmission, even in immunosuppressed animals.

We confirmed that the same parasite was present in both, insects and mouse lesions through ITS- 1 gene phylogeny tree. Thus, immunosuppression of the uninfected animals was able to demonstrate the competence of the vector Lu. longipalpis to transmit $L$. (L.) amazonensis to one of the two immunosuppressed mice. However, more studies using immunosuppressed animals are needed to better understand the relationship between the parasite and the host's weakened immune system.

We highlight a study ${ }^{34}$ in which the authors suggested the transmission of $L$. (L.) amazonensis to naïve (and immunocompetent) hamsters by the bite of wild Lu. longipalpis naturally infected by $L$. (L.) amazonensis. However, the hamster manifested clinical signs of VL 
only after three months, a behavior characteristic of $L$. (L.) infantum. These results differed from the ones of our study, in which the mouse developed skin lesions in less than one month. Moreover, two months after the infected insect bite, the disease has spread to the other paw, snout, and to the animal's internal organs (spleen, liver, and inguinal lymph nodes), the latter less common in CL.

Some studies point to a higher frequency of disseminated cutaneous manifestation in patients with coinfections, including reports of visceralization of the disease in both, humans $s^{35,36}$ and dogs ${ }^{17,37}$. Other studies have shown that the genetic variation of $L$. (L.) amazonensis strains has been related to the parasite tropism, in addition to causing a variety of clinical manifestations $\mathrm{s}^{35,38}$. Noteworthy, in the last decade, there have been changes in the profile of CL/AIDS coinfections, a fact justified mainly by the existence of a spatial overlap in the occurrence of the two diseases, with significant impact on the patient's survival, with increased risk of severe and widespread $\mathrm{CL}^{11,36}$. The Brazilian Ministry of Health recommends that the health secretaries of the States, including Maranhao, should increase the epidemiological surveillance of the two diseases, AIDS and leishmaniases ${ }^{39}$.

Considering that the natural vector of $L$. (L.) amazonensis is primarily wild (B. flaviscutellata), we reinforce that it can be replaced by another abundant species in the urban area, in this case, Lu. longipalpis, which can behave as a secondary vector for the parasite. Our study corroborates the one by Nogueira et al. ${ }^{4}$, who demonstrated that $L$. $(L$.) amazonensis completes its development to the metacyclic forms in Lu. longipalpis. Interestingly, in an endemic area for CL in central Colombia, Lu. longipalpis was reported but no cases of VL were reported in this $\operatorname{area}^{40}$. Another study carried out in a community of Panama in which the environment has undergone urbanization, presenting areas degraded by deforestation, Lu. longipalpis was also reported in an area endemic for $\mathrm{CL}^{16}$. Other studies have also mentioned rodents ${ }^{12}$ and dogs ${ }^{17}$ naturally infected by $L$. (L.) amazonensis in endemic areas for VL in Brazil.

In conclusion, our data demonstrated the competence of Lu. longipalpis to transmit $L$. (L.) amazonensis to an immunosuppressed mouse, causing leishmaniotic lesions. This finding highlights the epidemiological potential of $L u$. longipalpis for the occurrence of CL, especially in regions where the proven vectors are rare. We emphasize that both, Lu. longipalpis and L. (L.) amazonensis, coexist extensively in the Americas, including several regions of Brazil, where patients presenting HIV coinfection, using immunosuppressive drugs, or having comorbidities associated with malnutrition and socialeconomic problems are more vulnerable to the development of leishmaniasis.

\section{ACKNOWLEDGMENTS}

We are thankful to Dr. Mauricio Roberto Viana Sant'Anna, from the Parasitology Laboratory of the Federal University of Minas Gerais for providing L. amazonensis (IFLA/BR/1968/PH8).

\section{AUTHORS' CONTRIBUTIONS}

RCRS, SRFP and JMMR: conceptualization; JMMR and SRFP: project administration and funding acquisition; RCRS, LNPDC, JMSC and CEFA: sample collection and methodology; RCRS, SRFP and JMMR: formal analysis; RCRS, SRFP and JMMR: writing original draft preparation; RCRS and SRFP: writing review and editing. All authors have read and agreed to the published version of the manuscript.

\section{CONFLICT OF INTERESTS}

The authors declare that there have no conflict of interests.

\section{FUNDING}

This study was supported by Fundação de Amparo à Pesquisa e ao Desenvolvimento Científico e Tecnológico do Maranhão (FAPEMA No 00967-17) and by Comissão de Aperfeiçoamento de Pessoal do Nível Superior (CAPES) for providing the scholarship to RCRS (code 001).

\section{REFERENCES}

1. Pan American Health Organization. World Health Organization. Manual of procedures for leishmaniases surveillance and control in the Americas. Washington: PAHO; 2019.

2. Guimarães-e-Silva AS, Silva SO, Silva RC, Pinheiro VC, Rebêlo JM, Melo MN. Leishmania infection and blood food sources of phlebotomines in an area of Brazil endemic for visceral and tegumentary leishmaniasis. PLoS One. 2017;12:e0179052.

3. Fonteles RS, Pereira Filho AA, Moraes JL, Pereira SR, Rodrigues BL, Rebêlo JM. Detection of Leishmania DNA and blood meal identification in sand flies (Diptera: Psychodidae) from Lençois Maranhenses national park region, Brazil. J Med Entomol. 2018;55:445-51.

4. Nogueira PM, Guimarães AC, Assis RR, Sadlova J, Myskova J, Pruzinova K, et al. Lipophosphoglycan polymorphisms do not affect Leishmania amazonensis development in the permissive vectors Lutzomyia migonei and Lutzomyia longipalpis. Parasit Vectors. 2017;10:608.

5. Alexandre J, Sadlova J, Lestinova T, Vojtkova B, Jancarova M, Podesvova L, et al. Experimental infections and co-infections 
with Leishmania braziliensis and Leishmania infantum in two sand fly species, Lutzomyia migonei and Lutzomyia longipalpis. Sci Rep. 2020;10:3566.

6. Brazil RP, Rodrigues AA, Andrade Filho JD. Sand fly vectors of Leishmania in the Americas: a mini review. Entomol Ornithol Herpetol. 2015;4:144.

7. Alencar BF, Figueiredo IA. Perfil epidemiológico dos casos de Leishmaniose Tegumentar Americana no estado do Maranhão no período de 2015 a 2017. Rev Investig Biom. 2019;10:24350.

8. Cunha MA, Celeste BJ, Kesper N, Fugimori M, Lago MM, Ibanes AS, et al. Frequency of Leishmania spp. infection among HIV-infected patients living in an urban area in Brazil: a crosssectional study. BMC Infect Dis. 2020;20:885.

9. Machado CA, Sevá AP, Silva AA, Horta MC. Epidemiological profile and lethality of visceral leishmaniasis/human immunodeficiency virus co-infection in an endemic area in Northeast Brazil. Rev Soc Bras Med Trop. 2021;54:e07952020.

10. Oliveira RD, Pimentel KB, Magalhães FJ, Nascimento GC, Santos LL, Barros LA, et al. Ocorrência da coinfecção leishmaniose tegumentar americana/HIV no Estado do Maranhão. Rev Eletron Acervo Saude. 2019;11:e487.

11. Soares GH, Silva AB, Ferreira LS, Ithamar JS, Medeiros G, Pereira SR, et al. Case report: coinfection by Leishmania amazonensis and HIV in a Brazilian diffuse cutaneous leishmaniasis patient. Am J Trop Med Hyg. 2020;103:1076-80.

12. Caldart ET, Freire RL, Ferreira FP, Ruffolo BB, Sbeghen MR, Mareze M, et al. Leishmania in synanthropic rodents ( Rattus rattus ): new evidence for the urbanization of Leishmania. Braz J Vet Parasitol. 2017;26:17-27.

13. Carneiro FR, Amin GA, Cruz LB, Daher BA. Urban American cutaneous leishmaniasis. 2018;93:156-8.

14. Diniz MM, Ovallos FG, Gomes CM, Lavitschka CO, Galati EA. Host-biting rate and susceptibility of some suspected vectors to Leishmania braziliensis. Parasit Vectors. 2014;7:139.

15. Silva RC, Silva AS, Sousa SS, Bezerra JM, Rebêlo JM, Pinheiro VC. Occurrence of Phlebotominae (Diptera: Psychodidae) in urban leishmaniasis transmission foci in North-Eastern Brazil. J Med Entomol. 2019;56:247-53.

16. ValderramaA, Tavares MG, Andrade Filho JD. Report of Lutzomyia longipalpis (Lutz \& Neiva, 1912) (Diptera: Psychodidae: Phlebotominae) in a cutaneous-leishmaniasis-endemic area of Panama. Mem Inst Oswaldo Cruz. 2011;106:1049-51.

17. Valdivia HO, Almeida LV, Roatt BM, Reis-Cunha JL, Pereira AA, Gontijo C, et al. Comparative genomics of canine-isolated Leishmania (Leishmania) amazonensis from an endemic focus of visceral leishmaniasis in Governador Valadares, southeastern Brazil. Sci Rep. 2017;7:40804

18. Coelho MV, Falcão AR. Transmissão exeprimental de Leishmania braziliensis. II. Transmissão de amostra mexicana por picada de Phlebotomus longipalpis e de Phlebotomus renei. Rev Inst Med Trop Sao Paulo. 1962;4:220-4.

19. Rogers ME, Ilg T, Nikolaev AV, Ferguson MA, Bates PA. Transmission of cutaneous leishmaniasis by sand flies is enhanced by regurgitation of fPPG. Nature. 2004;430:463-7.

20. Cecílio P, Pires AC, Valenzuela JG, Pimenta PF, Cordeiro-da-Silva A, Secundino NF, et al. Exploring Lutzomyia longipalpis sand flies vector competence to Leishmania major parasites. J Infect Dis. 2020;222:1199-203.

21. Silva RC, Cruz LN, Coutinho JM, Santana NC, Rebêlo JM. Maintenance and productivity of a Lutzomyia longipalpis (Diptera: Psychodidae) colony from an area endemic for Visceral and Cutaneous Leishmaniasis in Northeastern Brazil. J Med Entomol. 2021;58:1917-25.

22. Pereira NC, Régis WC, Costa LE, Oliveira JS, Silva AG, Martins VT, et al. Evaluation of adjuvant activity of fractions derived from Agaricus blazei, when in association with the recombinant LiHyp1 protein, to protect against visceral leishmaniasis. Exp Parasitol. 2015;153:180-90.

23. Bin-Hafeez B, Ahmad I, Haque R, Raisuddin S. Protective effect of Cassia occidentalis L. on cyclophosphamide-induced suppression of humoral immunity in mice. J Ethnopharmacol. 2001;75:13-8

24. Schönian G, Nasereddin A, Dinse N, Schweynoch C, Schallig HD, Presber W, et al. PCR diagnosis and characterization of Leishmania in local and imported clinical samples. Diagn Microbiol Infect Dis. 2003;47:349-58.

25. Kumar S, Stecher G, Li M, Knyaz C, Tamura K. MEGA X: Molecular evolutionary genetics analysis across computing platforms. Mol Biol Evol. 2018;35:1547-9.

26. Castelo-Branco PV, Alves HJ, Pontes RL, Maciel-Silva VL, Pereira SR. Ascorbic acid reduces the genetic damage caused by miltefosine (hexadecylphosphocholine) in animals infected by Leishmania (Leishamnia) infantum without decreasing its antileishmanial activity. Int J Parasitol Drugs Drug Resist. 2019;9:8-15

27. Rodrigues OR, Marques C, Soares-Clemente M, Ferronha MH, Santos-Gomes GM. Identification of regulatory T cells during experimental Leishmania infantum infection. Immunobiology. 2009;214:101-11.

28. Tafuri WL, Santos RD, Arantes RM, Gonçalves R, Melo MN, Michalick MS, et al. An alternative immunohistochemical method for detecting Leishmania amastigotes in paraffinembedded canine tissues. J Immunol Methods. 2004;292:1723.

29. Pereira Filho AA, Bandeira MC, Fonteles RS, Moraes JL, Lopes CR, Melo MN, et al. An ecological study of sand flies (Diptera: Psychodidae) in the vicinity of Lençóis Maranhenses national park, Maranhão, Brazil. Parasit Vectors. 2015;8:442

30. Sousa-Gomes ML, Maia-Elkhoury AN, Pelissari DM, Lima Junior FE, Sena JM, Cechinel MP. Coinfecção Leishmania-HIV no 
Brasil: aspectos epidemiológicos, clínicos e laboratoriais. Epidemiol Serv Saude. 2011;20:519-26.

31. Akuffo H, Costa C, van Griensven J, Burza S, Moreno J, Herrero M. New insights into leishmaniasis in the immunosuppressed. PLoS Negl Trop Dis. 2018;12:e0006375.

32. Killick-Kendrick R, Rioux JA. Mark-release-recapture of sand flies fed on leishmanial dogs: the natural life-cycle of Leishmania infantum in Phlebotomus ariasi. Parassitologia. 2002;44:67-71.

33. Oliveira EF, Oshiro ET, Fernandes WS, Murat PG, Medeiros MJ, Souza AI, et al. Experimental infection and transmission of Leishmania by Lutzomyia cruzi (Diptera: Psychodidae): aspects of the ecology of parasite-vector interactions. PLoS Negl Trop Dis. 2017; 11:e005401.

34. Sherlock IA. Ecological interactions of visceral leishmaniasis in the State of Bahia, Brazil. Mem Inst Oswaldo Cruz. 1996;91:671-83.

35. Barral A, Pedral-Sampaio D, Grimaldi G, Momen H, McMahonPratt D, Ribeiro de Jesus A, et al. Leishmaniasis in Bahia, Brazil: evidence that Leishmania amazonensis produces a wide spectrum of clinical disease. Am J Trop Med Hyg. 1991;44:536-46.

36. Zijlstra EE. PKDL and other dermal lesions in HIV co-infected patients with leishmaniasis: review of clinical presentation in relation to immune responses. PLoS Negl Trop Dis. 2014;8: e3258.
37. Tolezano JE, Uliana SR, Taniguchi HH, Araújo MF, Barbosa JA, Barbosa JE, et al. The first records of Leishmania (Leishmania) amazonensis in dogs (Canis familiaris) diagnosed clinically as having canine visceral leishmaniasis from Araçatuba County, São Paulo State, Brazil. Vet Parasitol. 2007;149:280-4.

38. Oliveira JP, Fernandes F, Cruz AK, Trombela V, Monteiro E, Camargo AA, et al. Genetic diversity of Leishmania amazonensis strains isolated in northeastern Brazil as revealed by DNA sequencing, PCR-based analyses and molecular karyotyping. Kinetoplastid Biol Dis. 2007;6:5.

39. Brasil. Ministério da Saúde. Secretaria de Vigilância em Saúde. Departamento de Vigilância Epidemiológica. Manual de recomendações para diagnóstico, tratamento e acompanhamento da co-infecção Leishmania-HIV. Brasília: Ministério da Saúde; 2011.

40. López Y, Osorio L, Alvarez G, Rojas J, Jiménez F, Gómez C, et al. Sandfly Lutzomyia longipalpis in a cutaneous leishmaniasis focus in Central Colombia. Mem Inst Oswaldo Cruz. 1996;91:415-9.

Supplementary Material available from: https://doi.org/10.48331/scielodata.JRNFAZ 